\title{
Multi-bunch Dynamics in Accelerating Structures Including Interaction with Higher Order Modes.
}

\author{
M.Ferrario *, L.Serafini \#, F. Tazzioli * \\ * INFN Laboratori Nazionali di Frascati \\ \# INFN Sezione di Milano
}

\begin{abstract}
The case of interaction of not fully relativistic multibunch beams with the fundamental and higher order modes of a cavity is not yet covered by existing codes, nevertheless it is a fundamental problem in the design of RF guns and/or collider cavities. A simple model that couples Newton and Maxwell equations, taking into account also space charge, beam loading and build-up effects of higher order modes under beam-tube cutoff frequency, is presented. This approach is intended to fill that gap, avoiding relativistic approximation. It uses a current density description of the beam and a slowly varying envelope approximation for the time evolution of the modes amplitude. A fast running code (HOMDYN), based on this model has been developed and the application to a few typical examples is illustrated.
\end{abstract}

Table 1

Symbol definitions

\begin{tabular}{|l|l|}
\hline $\mathrm{A}_{\mathrm{n}}=$ mode amplitude & $\mathrm{L}_{\mathrm{b}}=$ bunch length \\
$\hat{\mathrm{e}}_{\mathrm{n}}=$ mode spatial distri- & $\mathrm{q}=$ bunch charge \\
bution on axis & $\mathrm{z}_{\mathrm{h}, \mathrm{t}, \mathrm{b}=\text { head, tail and bary- }}$ \\
$\omega_{\mathrm{n}}=$ mode radian frequency & center coordinates \\
$\phi_{\mathrm{n}}=$ mode phase & $\mathrm{R}=$ bunch radius \\
$\alpha_{\mathrm{n}}=$ mode complex ampli- & $\mathrm{C}=$ light velocity \\
tude & $\mathrm{m}_{\mathrm{o}}=$ clectron rest mas \\
$\mathrm{Q}_{\mathrm{n}}=$ quality factor of the & $B=$ avcrage velocity/light \\
mode & velocity \\
$\mathrm{U}_{\mathrm{n}}=$ mode stored energy & $\mathrm{J}=$ beam current density \\
$\mathrm{E}_{\mathrm{O}}=$ peak electric field & $\mathrm{N}_{\mathrm{b}}=\mathrm{n}$. of bunches in train \\
C.C.= Complex Conjugate & $\mathrm{v}_{\mathrm{r}}=$ repetition rate \\
\hline
\end{tabular}

\section{INTRODUCTION}

When treating the evolution of high charge, not fully relativistic electron bunches in RF fields of an accelerating cavity, it is necessary to take into account also the field induced by the beam in the fundamental and higher order modes, and the variation of bunch sizes due to both the external fields and space charge.

For single bunches the problem has been already tackled using PIC codes, which describe the bunch as an ensemble of particles and track their motion, coupled to the E.M. field propagation. The case of long bunch trains would consume an unbearable amount of computer time if treated by a mere extension of the single bunch case.

We have therefore devised a simple model that uses a current density description of the beam and slowly varying envelope approximation (SVEA) for the cvolution of the cavity modes. The present version deals only with TM monopole modes: an extended version comprehensive of dipole modes is under development.

The SVEA approximation supposes small field perturbations produced by any single bunch, that add up to give an envelope of any mode field slowly varying on the time scale of its period.

Motion and field equations are coupled together through the driving current term.

\section{THE FIELD EQUATIONS}

Expressing the electric field $E$ as a sum of normal orthogonal modes:

$$
\begin{array}{r}
E(z, t)=\sum_{n} A_{n}(t) \hat{e}_{n}(z) \sin \left(\omega_{n} t+\phi_{n}(t)\right) \\
=\sum_{n}\left(\alpha_{n} e^{i \omega_{n} t}+\alpha_{n}^{*} e^{-i \omega_{n} t}\right) \hat{e}_{n}(z) \\
\text { with } \alpha_{n}=\left(A_{n} / 2 i\right) e^{i \phi_{n}}, \hat{e}_{n}(z)=\hat{e}_{n}(r=0) \text { and: } \\
\nabla \hat{e}_{n}=-k_{n}^{2} \hat{e}_{n} \int_{v} \hat{\mathbf{e}}_{n} \hat{\mathbf{e}}_{m} d v=\delta_{n m}
\end{array}
$$

the cquation for the electric field complex amplitude $\alpha_{n}$ driven by a beam current distribution $J(z, t)$ in the cavity is [1]:

$$
\begin{aligned}
\frac{d^{2} \alpha_{n}}{d^{2}} & +\left(2 i \omega_{n}+\frac{\omega_{n}}{Q_{n}}\right) \frac{d \alpha_{n}}{d t}+i \frac{\omega_{n}^{2}}{Q_{n}} \alpha_{n}= \\
& =-\frac{e^{-i \omega_{n}}}{\varepsilon} \int_{v}\left(\frac{\partial J}{\partial t} \cdot \hat{e}_{n}\right) d v
\end{aligned}
$$

with the normalization relations:

$$
\left|\alpha_{n}(t)\right|=\sqrt{\frac{U_{n}(t)}{2 \varepsilon}} \quad \hat{e}_{n}(z)=\sqrt{\frac{\varepsilon}{2 U_{n, o}}} \widehat{E}_{n, o}(z)
$$

Applying the SVEA approximation hypotheses:

$$
\frac{\mathrm{d} \alpha_{\mathrm{n}}}{\mathrm{dt}} \ll \omega_{\mathrm{n}} \alpha_{\mathrm{n}} \quad \frac{\mathrm{d}^{2} \alpha_{\mathrm{n}}}{\mathrm{dt}^{2}}<\omega_{\mathrm{n}}^{2} \alpha_{\mathrm{n}}
$$

we obtain the first order equation:

$$
\begin{gathered}
\frac{\mathrm{d} \alpha_{n}}{d t}+\left(1+\frac{i}{2 Q_{n}}\right) \frac{\omega_{n}}{2 Q_{n}} \alpha_{n}= \\
=i \frac{e^{-i \omega_{n}}}{2 \omega_{n} \varepsilon}\left(1+\frac{i}{2 Q_{n}}\right) \int_{v}\left(\frac{\partial J}{\partial t} \cdot \hat{e}_{n}\right) d v+
\end{gathered}
$$




$$
+\left(1+\frac{\mathrm{i}}{2 \mathrm{Q}_{1}}\right) \frac{2 \omega_{1}}{\mathrm{Q}_{1}} \mathrm{~K}_{1}
$$

The last term is effective only in the fundamental mode equation and accounts for a feeding sinusoidal current of amplitude $\mathrm{K}_{1}$ representing a power supply.

$$
\int_{V}\left(\frac{\partial \mathrm{J}}{\partial \mathrm{t}} \cdot \hat{\mathrm{e}}_{\mathrm{n}}\right) \mathrm{dv}=\frac{\mathrm{q} \beta \mathrm{c}}{\mathrm{L}_{\mathrm{b}}}\left(\hat{\mathrm{e}}_{\mathrm{n}}\left(\mathrm{z}_{\mathrm{h}}\right) \frac{\mathrm{dz}}{\mathrm{dt}}-\hat{\mathrm{e}}_{\mathrm{n}}\left(\mathrm{z}_{\mathrm{t}}\right) \frac{\mathrm{d} \mathrm{z}_{\mathrm{t}}}{\mathrm{dt}}\right)
$$

The evolution of the field amplitude during the bunch to bunch interval is given by the analytical solution of the homogeneous equation, which connects successive numerical integrations applicd during any bunch transit.

\section{THE BEAM EQUATIONS}

The basic approximation in the description of beam dynamics lays in the assumption that each bunch is represented as a uniform charged cylinder, whose length and radius can vary under a self-similar time evolution, i.e. keeping anyway uniform the charge distribution inside the bunch. The present choice of a uniform distribution is dictatcd just by sake of simplicity in the calculation of space charge and HOM contributions to the beam dynamics. A further improvement of the model to include gaussian distributed bunches is under way. According to this assumption, and to the general hypothesis that the space charge and HOM effects on beam dynamics are perturbative, we can write, under a paraxial approximation, the equations for the longitudinal motion of the bunch barycenter:

$$
\frac{d \beta}{d t}=\frac{e}{m_{o} c \gamma} \sum_{n}\left(\alpha_{n} e^{i \omega_{n} t}+\alpha_{n}^{*} e^{-i \omega_{n} t}\right) \hat{e}_{n}(z) \quad \frac{d z_{b}}{d t}=\beta c
$$

The evolution of the bunch radius $\mathrm{R}$ is described according to a recently proposed envelope equation [2], including RF-focusing and space charge effects, transformed into the time-domain:

$$
\begin{gathered}
\frac{\mathrm{d}^{2} \mathrm{R}}{\mathrm{dt}{ }^{2}}+\frac{\mathrm{dR}}{\mathrm{dt}} \frac{\mathrm{d} \beta}{\mathrm{dt}} \mathrm{g}(\beta)+\frac{\mathrm{R}}{4 \gamma^{2}}\left(\left(\frac{\mathrm{dR}}{\mathrm{dt}}\right)^{2} \mathrm{f}(\beta)+\mathrm{K}_{\mathrm{r}}\right)+ \\
-\frac{\mathrm{Ic} \mathrm{c}^{2}}{\mathrm{I}_{\mathrm{A}} \mathrm{R} \beta \gamma^{3}}-\frac{\varepsilon_{\mathrm{n}} \mathrm{c}^{2}}{\mathrm{R}^{3} \gamma^{2}}=0
\end{gathered}
$$

$g(\beta)=\frac{1-\beta \gamma^{2}-1 / \gamma}{\beta} f(\beta)=\frac{1}{\beta^{2}}\left(3 \beta^{2} \gamma^{2}-2 \gamma^{3}(1+\beta)+\gamma^{4}-1-\frac{2}{\beta}\left(1-\beta^{2} \gamma^{2}\right)\right)$

where $I_{A}$ is the Alfven current $(17 \mathrm{kA}), \varepsilon_{n}$ the rms normalized beam emittance and the RF average focusing gradient $K_{r}$ is given by:

$$
\begin{aligned}
K_{r} & =\sum_{n}\left(\frac { 2 e ^ { 2 } | \alpha | _ { n } ^ { 2 } } { m _ { o } c ^ { 2 } } \sum _ { 2 } ^ { \infty } \left(a_{s+1, n}^{2}+a_{s-1, n}^{2}+\right.\right. \\
& \left.+2 a_{s+1, n} a_{s-1, n} \cos \left(2\left(\phi_{n}(t)-\phi_{n, o}\right)\right)\right)
\end{aligned}
$$

where the two sums run over the HOM modes (index $n, n=1$ for the fundamental mode) and over the spatial harmonic coefficients $a_{s, n}$ of each mode form factor $\hat{e}_{n}(z)=\Sigma_{n} a_{s, n} \cos \left(n k_{n} z\right)$.

The bunch lengthening is derived adding to the space charge effect, as given by [3], the first order component coming from fundamental and HOM modes, which is simply given by the head-tail difference of the total RF field acting on the bunch:

$$
\begin{gathered}
\frac{\mathrm{d}^{2} \mathrm{~L}_{\mathrm{b}}}{\mathrm{dt}^{2}}=\frac{\mathrm{eq}}{2 \varepsilon_{\mathrm{o}} \mathrm{m}_{\mathrm{o}} \mathrm{R}^{2} \mathrm{~L}_{\mathrm{b}}}\left(\sqrt{\left(\gamma \mathrm{L}_{\mathrm{b}}\right)^{2}+\mathrm{R}^{2}}-\left(\gamma \mathrm{L}_{\mathrm{b}}+\mathrm{R}\right)\right)+ \\
+\frac{\mathrm{e}}{3} \sum_{\mathrm{n}}\left(\alpha_{v}(\mathrm{t}) \mathrm{e}^{\mathrm{i} \omega_{\mathrm{n}} \mathrm{t}}\left(\hat{\mathrm{e}}_{\mathrm{n}}\left(\mathrm{z}_{\mathrm{h}}\right)-\hat{\mathrm{e}}_{\mathrm{n}}\left(\mathrm{z}_{\mathrm{t}}\right)\right)+\text { C.C. }\right) \\
\mathrm{m}_{\mathrm{o}}{ }^{3}
\end{gathered}
$$

In a similar way we derive the energy distribution inside the bunch by specifying the energy associated to each slice located at a distance $s$ from the bunch barycenter $\left(z_{b}\right)$ :

$$
\begin{gathered}
\frac{\mathrm{d} \gamma_{\mathrm{s}}}{\mathrm{dt}}=\frac{\mathrm{e}}{\mathrm{m}_{\mathrm{o}} \mathrm{c}} \beta_{\mathrm{s}}\left(\sum_{\mathrm{n}}\left(\alpha_{\mathrm{n}}(\mathrm{t}) \mathrm{e}^{\mathrm{i} \omega_{\mathrm{n}} \mathrm{l}} \hat{\mathrm{e}}_{\mathrm{n}}\left(\mathrm{z}_{\mathrm{b}}+\mathrm{s}\right)+\mathrm{C} . \mathrm{C} .\right)+\right. \\
+\frac{\mathrm{q} \mathrm{s}}{\varepsilon_{\mathrm{o}} \gamma \mathrm{R}^{2} \mathrm{~L}_{\mathrm{b}}^{2}}\left(\sqrt{\left(\gamma \mathrm{L}_{\mathrm{b}}\right)^{2}+\mathrm{R}^{2}}-\left(\gamma \mathrm{L}_{\mathrm{b}}+\mathrm{R}\right)\right)
\end{gathered}
$$

\section{FIRST RESULTS.}

To test the validity of the simulation we have applied the computation to the case of a $500 \mathrm{MHz}$ single cell resonator, computing the mode frequencies and field distributions by the SUPERFISH code. Starting with the case of a train of relativistic rigid bunches and assuming an extreme set of parameters to shorten the time scale of the phenomena $(\gamma=100$, $\mathrm{q}=400 \mathrm{nC}, \mathrm{Q}=10^{2}, \mathrm{~N}_{\mathrm{b}}=10, \mathrm{v}_{\mathrm{r}}=500 \mathrm{MHz}, \mathrm{U}_{010}=0.138 \mathrm{~J}$, $\mathrm{U}_{012}=0$. J) we show in Fig. 1 the evolution of the electric field seen by bunches during transit in the cavity. In Fig.2,3 the stored energies and field phases of $\mathrm{TM}_{010}$ are shown for two different injection phases. Notice the transition from absorption to emission regime after 3 bunches in the $\mathrm{TM}_{010}$ mode

Induced energy in $\mathrm{TM}_{010}$ (Fig.4) and in $\mathrm{TM}_{012}$ (Fig.5) are compared for a relativistic $(\gamma=5)$ and non-relativistic $(\gamma=2)$ beam, $\left(\mathrm{q}=16 \mathrm{nC}, \mathrm{Q}=10^{2}, \mathrm{~N}_{\mathrm{b}}=200, v_{\mathrm{r}}=125 \mathrm{MHz}, \mathrm{U}_{010}=0 \mathrm{~J}\right.$, $\mathrm{U}_{012}=0 \mathrm{~J}$ ). 


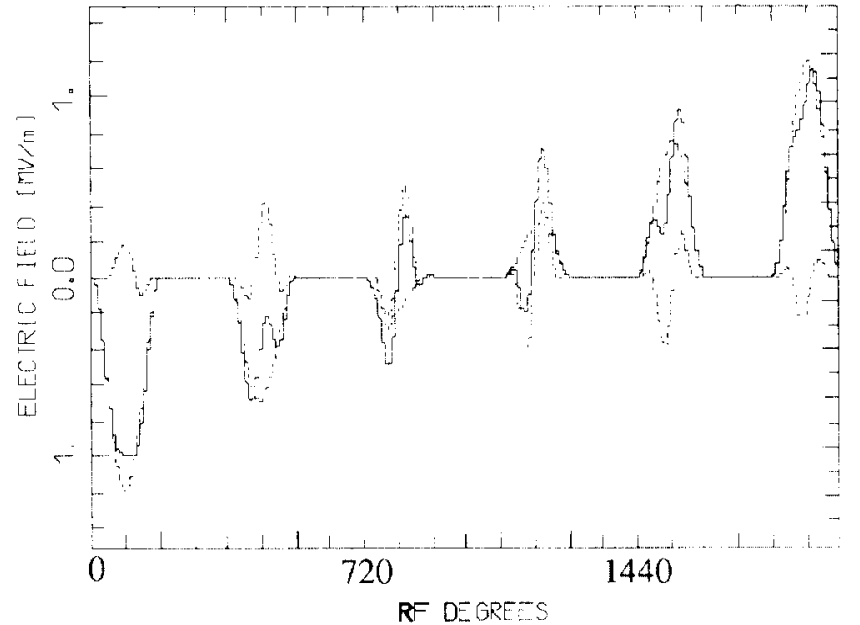

Figure 1 - Electric field seen by bunches, dashed lines are $\mathrm{TM}_{010}$ and $\mathrm{TM}_{012}$ fields, full line is the superposition of the two modes.

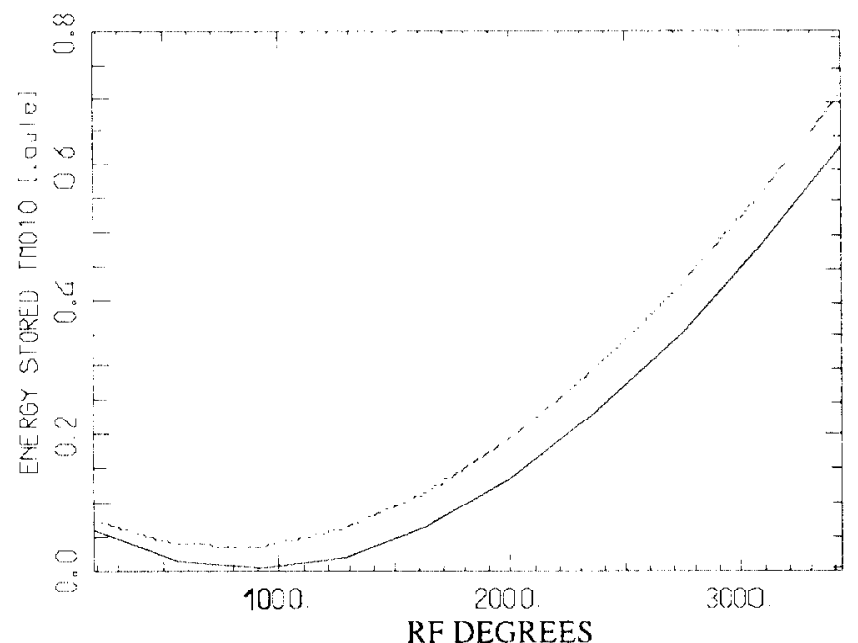

Figure 2 - Stored encrgies of $\mathrm{TM}_{010}$ for two different injection phases, full line $\phi_{0}=-90$, dashed line $\phi_{0}=-60$.

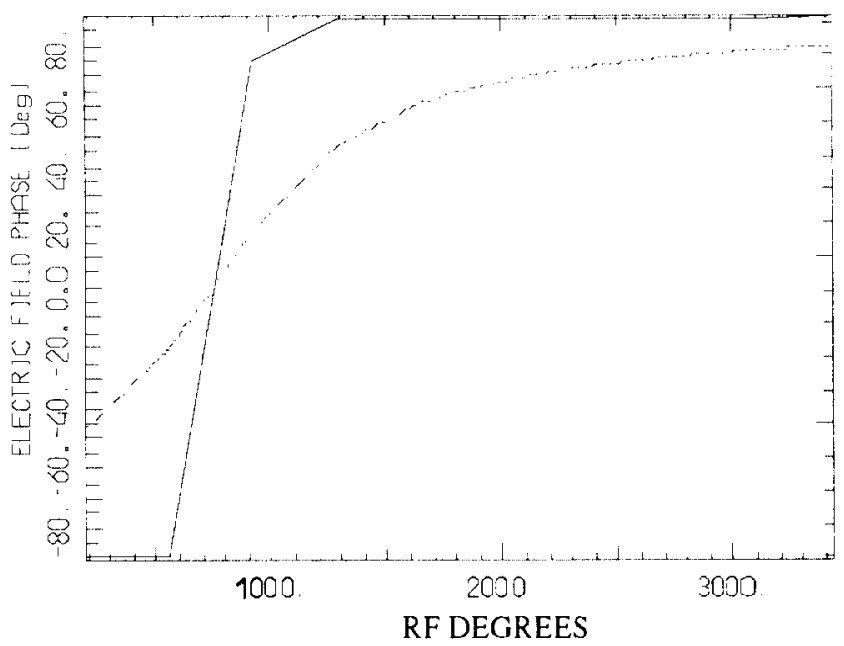

Figure 3 - TM010 phase evolution for two different injection phases, full line $\phi_{0}=-90$, dashed line $\phi_{0}=-60$.

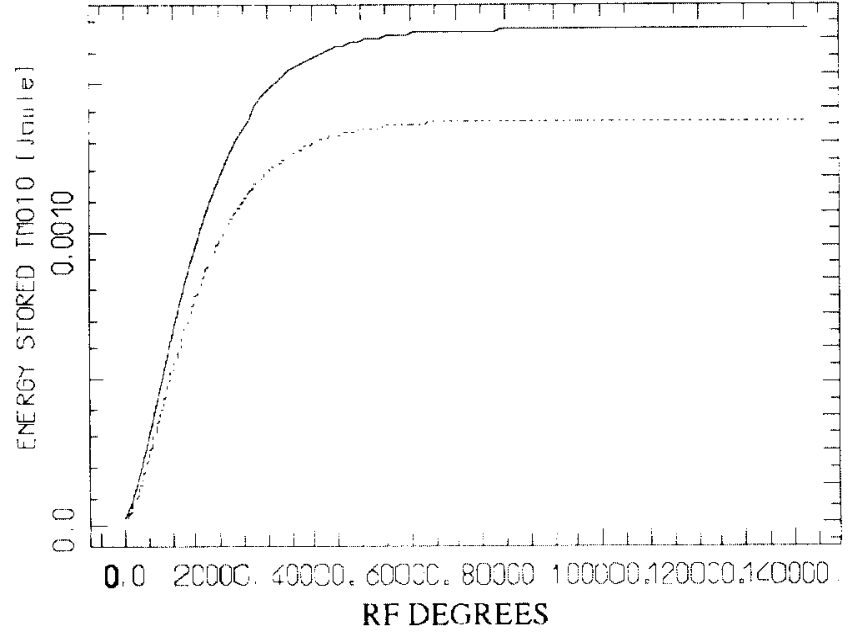

Figure 4 - Induced energy in $\mathrm{TM}_{010}$ is compared for a relativistic ( $\gamma=5$ full line) and non-relativistic ( $\gamma=2$ dashed line) beam.

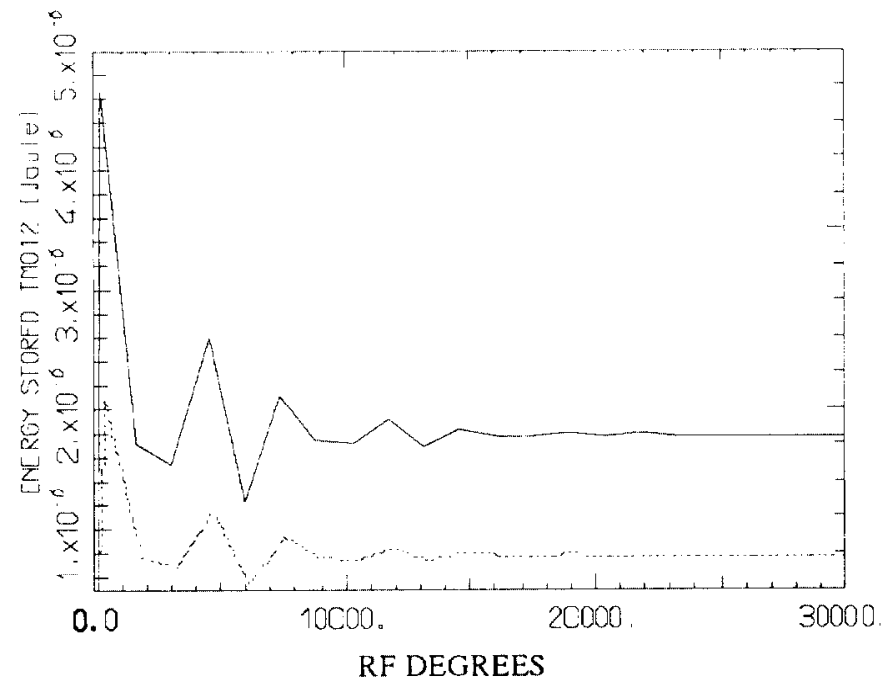

Figure 5 - Induced energy in $\mathrm{TM}_{012}$ is compared for a relativistic ( $\gamma=5$ full line) and non-relativistic ( $\gamma=2$ dashed line) beam. (RF Deg on the scale of $\mathrm{TM}_{010}$ period)

\section{REFERENCES}

[1] M. Puglisi, "Conventional RF cavity design," $C A S$ School, CERN 92-03, (1992).

[2] S.C. Hartman, J. B. Rosenzweig, "Ponderomotive focusing in axisymmetric RF linacs," Phys. Rev. E, Vol. 47, N. 3, (1993).

[3] G. Mavrogenes, "Space charge effects in high current linac transport systems," ANL Report. 